\title{
Starspot Activity of V711 Tau from November to December 2005
}

\author{
Sheng-hong $\mathrm{Gu}^{1} \dagger$, Andrew Collier Cameron ${ }^{2}$, \\ J.R. Barnes ${ }^{3}$, and Jia-yan Yang ${ }^{1}$ \\ ${ }^{1}$ National Astronomical Observatories/Yunnan Observatory, CAS, Kunming, \\ Yunnan Province, China \\ email: shenghonggu@ynao.ac.cn \\ ${ }^{2}$ SUPA, School of Physics and Astronomy, University of St. Andrews, North Haugh, \\ St. Andrews, Fife, UK \\ ${ }^{3}$ School of Physics, Astronomy and Mathematics, University of Hertfordshire, College Lane, \\ Hatfield, UK
}

\begin{abstract}
Two new high-resolution spectroscopic datasets of the active binary V711 Tau are analyzed here. Using the Least-Squares Deconvolution method, we have derived the time series profiles of V711 Tau with high signal-to-noise ratio, which have been used to reconstruct the starspot patterns for its two components by means of the Doppler imaging code DoTS. The results show that the high latitude spots are main features for the primary, the low latitude spots are clear for the secondary in both of two observing runs, and the starspot pattern changes obviously during one month. The lifetime of intermediate-low latitude spots is shorter than the high latitude spots for the primary, the low latitude spots of the secondary seem to have a longer lifetime.
\end{abstract}

Keywords. Stellar activity, Starspots, Doppler imaging

\section{Introduction}

Since Vogt \& Penrod (1983) obtained the first Doppler image of the RS CVn binary V711 Tau (K1IV+G5V), many investigators (e.g., Vogt et al. 1999, Donati 1999, Strassmeier \& Bartus 2000, Garcia-Alvarez et al. 2003, Donati et al. 2003, Petit et al. 2004) have studied this very active star using Doppler imaging technique. However, most of the Doppler image sets of V711 Tau have a low time resolution except the ones derived by Strassmeier \& Bartus (2000), so it is impossible to have any insight into the detailed starspot evolution. Here we describe the preliminary Doppler imaging of V711 Tau based on two datasets obtained at Xinglong station one month apart.

\section{Observation}

V711 Tau was observed at Xinglong Station of NAOC, China on 18-24 Nov 2005 and 17-22 Dec 2005 using the 2.16-m telescope and Coudé echelle spectrograph with a $1 \mathrm{k} \times 1 \mathrm{k}$ CCD detector (Zhao \& Li 2001). The wavelength coverage is $5796-9048 \AA$, with a resolution of 36000 . A total of 22 spectra for V711 Tau were obtained for the first observing run, and 10 spectra for the second one. The data reduction was done using the IRAF package according to the standard fashion.

$\dagger$ Present address: National Astronomical Observatories/Yunnan Observatory, CAS, Kunming, Yunnan Province, China 

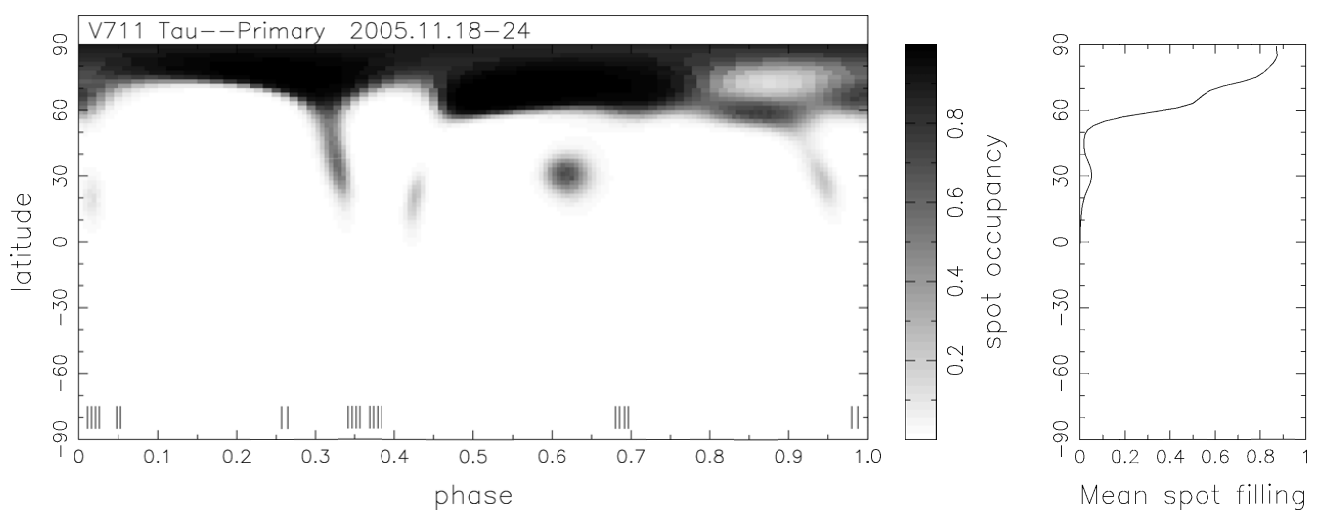

Figure 1. The Doppler image of the primary for V711 Tau on 18-24 Nov 2005.
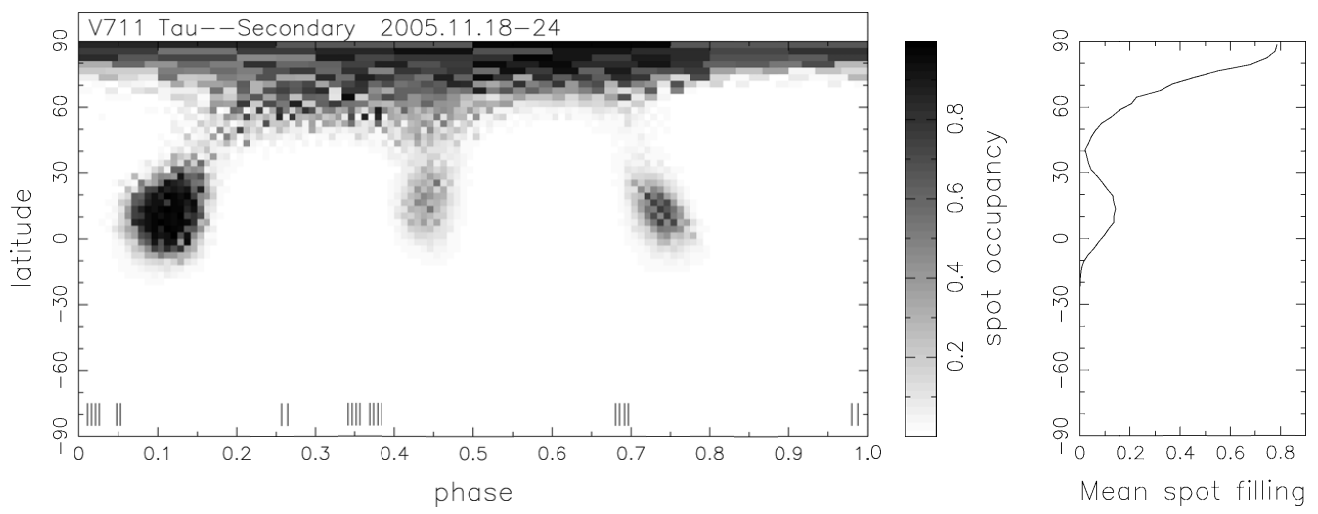

Figure 2. The Doppler image of the secondary for V711 Tau on 18-24 Nov 2005.
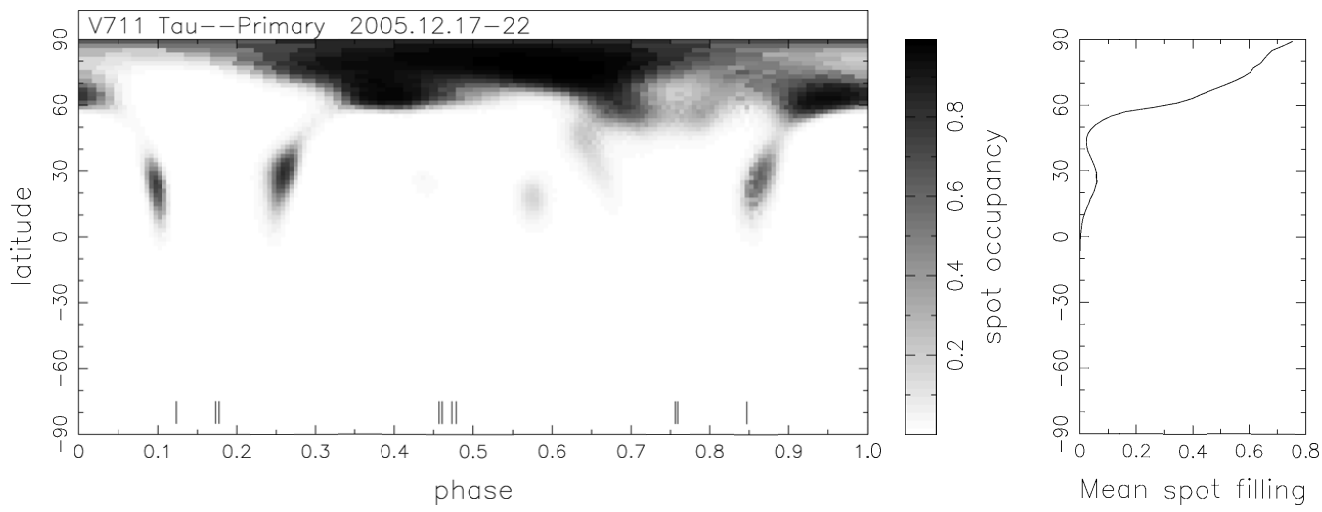

Figure 3. Same as Figure 1, but for 17-22 Dec 2005. 

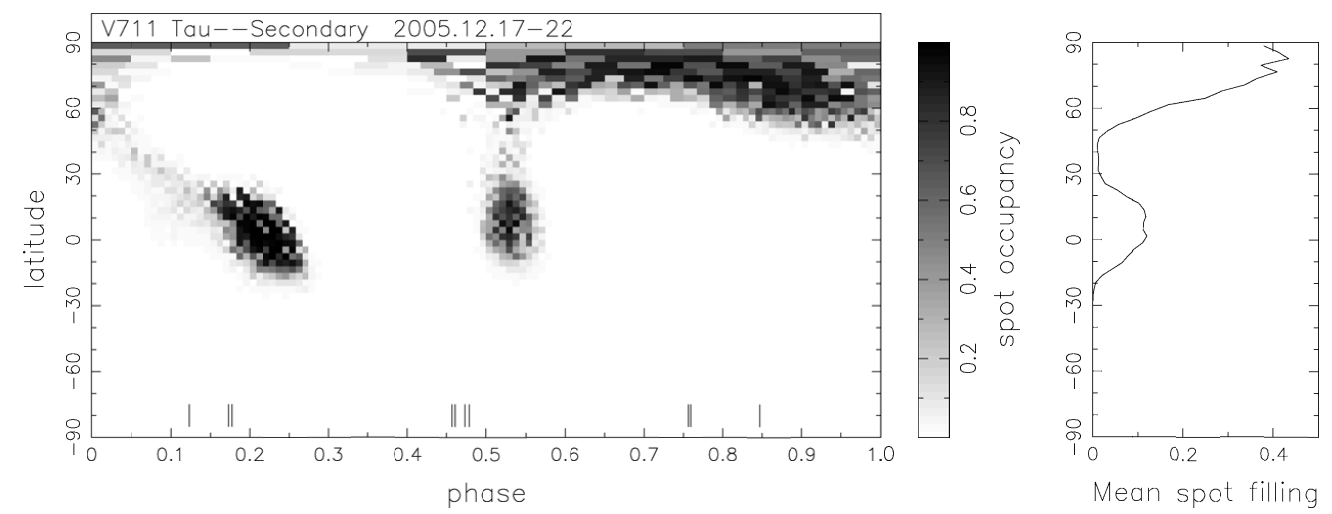

Figure 4. Same as Figure 2, but for 17-22 Dec 2005.

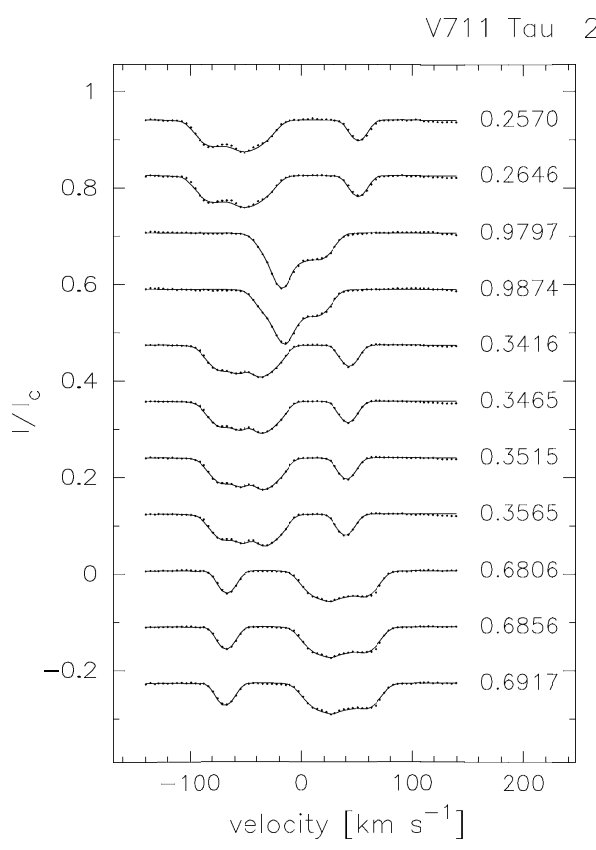

$2005.11 .18-24$

Figure 5. The LSD profiles and fitting on 18-24 Nov 2005.

\section{Image reconstruction}

We have used the binary mode of the Doppler imaging code DoTS (Collier Cameron 1997) to reconstruct the starspot patterns of the two components of V711 Tau. Before Doppler imaging, we used the Least-Squares Deconvolution (Donati et al. 1997) method to enhance the $\mathrm{S} / \mathrm{N}$ of the profiles. In the course of the Doppler imaging, we used the spectra of two template stars HR 4182 (K1IV) and HR 255 (G5IV) to mimic the photospheres of the primary and the secondary, and the spectrum of one template star HR 248 (M0III) to the spot. The final Doppler images based on the two datasets, and the relative profile fitting are shown in Figures 1-6. 


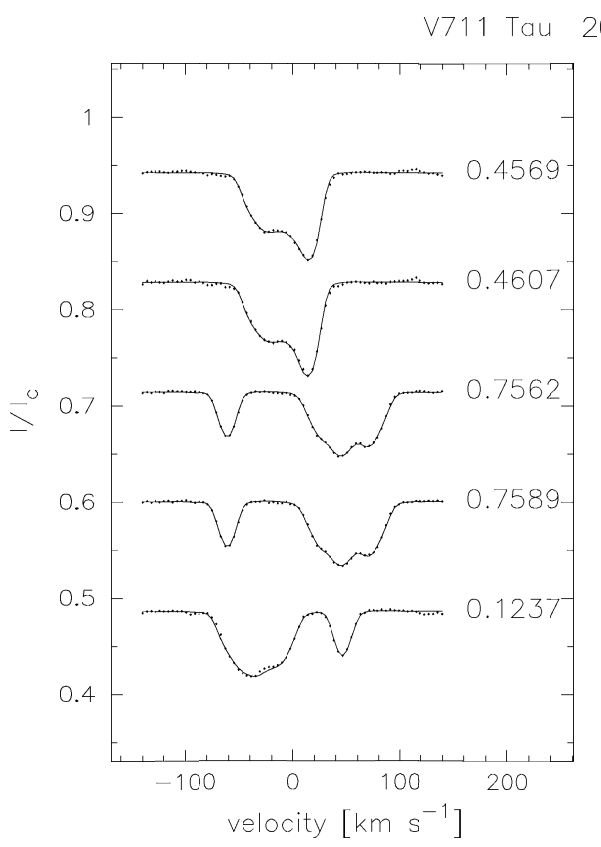

$2005.12 .17-22$

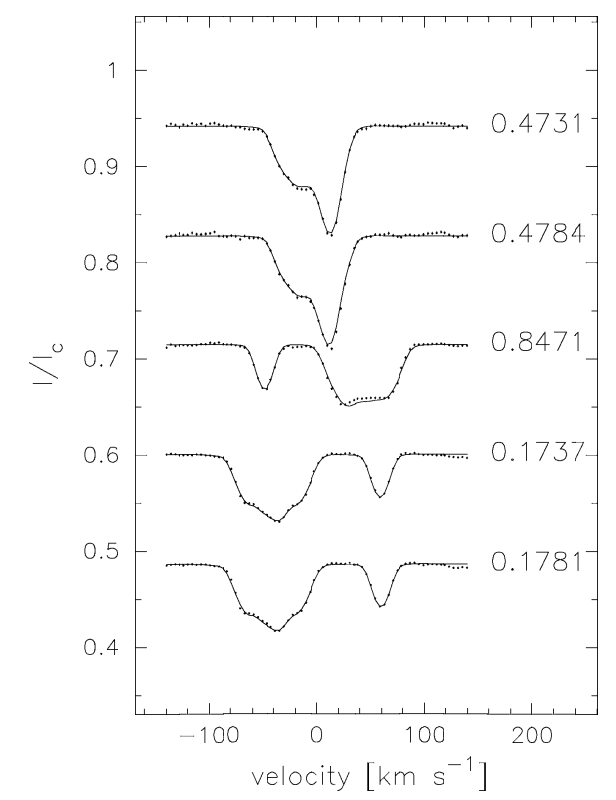

Figure 6. Same as Figure 5, but for 17-22 Dec 2005.

\section{Discussion and conclusions}

The Doppler imaging of V711 Tau described above demonstrates that there were some intermediate-low latitude spots besides the predominant polar spots during two observing runs. In Figures 1 and 3 we see that both the intermediate-low latitude spots and the polar spots for the primary changed during one month, especially for the intermediatelow latitude spots, which agrees with the results of Strassmeier \& Bartus (2000). It seems that two polar spots which appeared in November became closer to each other and smaller in December. As for the intermediate-low latitude spots, it's hard to determine how they evolved during one month. This means we need to do more dense observations to clarify it during next observing season, as was done by Strassmeier \& Bartus (2000). For the secondary of V711 Tau, the quality of Doppler imaging is not as good due to the low rotational velocity, but we can still derive some information about its starspot activity. For example, from Figures 2 and 4, it can be seen that two low-latitude spots at phases 0.1 and 0.44 in November migrate to phases 0.2 and 0.52 in December.

The preliminary Doppler images of V711 Tau from these two observing runs show that the main spot features of the primary are located in the high-latitude region, and there also are some intermediate-low latitude spots with lifetime shorter than one month. The main spot features of the secondary are located in low-latitude and equatorial regions, and it appears that the low-latitude spots have a longer lifetime than the ones on the primary.

\section{Acknowledgements}

We would like to thank Drs. Jian-yan Wei and Xiao-jun Jiang for supporting our research project in 2.16-m telescope of Xinglong station. This work is supported by NSFC under grant No. 10373023. 


\section{References}

Collier Cameron, A. 1997, MNRAS 287, 556

Donati, J.-F. et al. 1997, MNRAS 291, 658

Donati, J.-F. 1999, MNRAS 302, 457

Donati, J.-F. et al. 2003, MNRAS 345, 1145

Garcia-Alvarez, D. et al. 2003, A\&SA 402, 1073

Petit, P. et al. 2004, MNRAS 348, 1175

Strassmeier, K.G. \& Bartus, J. 2000, A\&A 354, 537

Vogt, S.S. \& Penrod, G.D. 1983, PASP 95, 565

Vogt, S.S. et al. 1999, ApJS 121, 547

Zhao, G. \& Li, H.-B. 2001, ChJAA 1, 555 